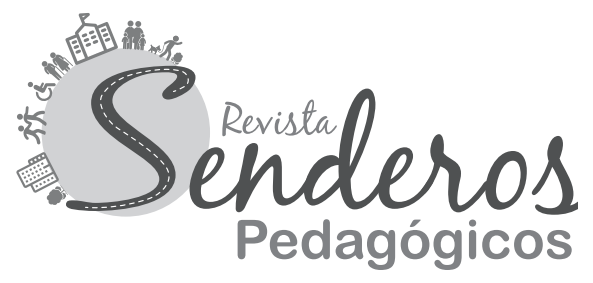

\title{
Práctịcas de cuidado implementadas por los niños, niñas, adolescentes y sus familias durante la enfermedad ${ }^{1}$
}

\author{
Care practices implemented by children, adolescents and \\ their families during the disease
}

\section{Autoras: \\ Manuela Arrubla Gutiérrez², Jennifer Giraldo Mesa ${ }^{3}$, Valentina Monsalve Mejía ${ }^{4}$, Magdalena Sánchez Vélez ${ }^{5}$, Nadia Milena Henao García ${ }^{5}$.}

Recibido: 01/07/2020

Aprobado: 28/10/2020
1 Artículo de investigación.

2 Licenciada en Educación Preescolar del Tecnológico de Antioquia, Institución Universitaria. manu2034@hotmail.com

3 Licenciada en Educación Preescolar del Tecnológico de Antioquia, Institución Universitaria. jeny.1016@hotmail.com

4 Licenciada en Educación Preescolar del Tecnológico de Antioquia, Institución Universitaria. valmonme@gmail.com

5 Licenciada en Educación Preescolar del Tecnológico de Antioquia, Institución Universitaria. magda9613@hotmail.com

6 Magíster en Educación de la Universidad de San Buenaventura Medellín, Licenciada en Educación Preescolar del Tecnológico de Antioquia, Institución Universitaria. Coordinadora de la línea de investigación sobre infancias del Grupo Senderos y de la Licenciatura en Educación Infantil del Tecnológico de Antioquia, Institución Universitaria.nmhenaog@tdea.edu.co, milenaho2@gmail.com.

\section{Resumen:}

El artículo presenta los resultados del proyecto investigativo y pedagógico sobre las prácticas de cuidado implementadas por las familias de niños, niñas $y$ adolescentes (NNA) con Enfermedades Crónicas No Transmisibles (ECNT), en tres instituciones de salud en Medellín, Colombia. Esta investigación cualitativa, desde la perspectiva interpretativa y con enfoque narrativo, implementó como técnica los relatos de vida; la propuesta pedagógica se sustentó desde el taller, cuyas actividades rectoras: juego y arte, se registraron en diarios pedagógicos. Los datos se sistematizaron en una matriz de análisis cuyas unidades de sentido, pre-categorías y categorías emergentes fueron: emociones, juego y experiencias de aprendizaje; proceso de cuidado durante la enfermedad; hábitos de cuidado $\mathrm{y}$ autocuidado durante la enfermedad; y aprendizajes respecto al cuidado ante la enfermedad. Entre los resultados más importantes están la identificación de cambios en las rutinas debido a la alteración de la salud y la implementación de nuevas prácticas de cuidado. 
Palabras clave: infancia, familia, prácticas, cuidados, enfermedad.

\begin{abstract}
The article presents the results of the research and pedagogical project on the care practices implemented by the families of children and adolescents (NNA, for its abbreviation in Spanish) with chronic non-communicable diseases (NCD) in three health institutions in Medellin, Colombia. This qualitative research, from an interpretative perspective and with a narrative approach, implemented life stories as a technique; the pedagogical proposal was supported from the workshop whose
\end{abstract}

guiding activities, play and art, were recorded in pedagogical journals. The data were systematized in an analysis matrix whose emerging units of meaning, pre-categories, and categories were: emotions, play and learning experiences; process of care during the disease; habits of care and self-care during the disease; and learning about care for the disease. Among the most important results are the identification of changes in routines due to health alteration and the implementation of new care practices.

Keywords: childhood, family, practices, care, disease.

\section{Introducción}

El proyecto investigativo y pedagógico surgió con el propósito de analizar las prácticas de cuidado implementadas por las familias de niños, niñas y adolescentes (NNA) con Enfermedades Crónicas No Transmisibles (ECNT). A partir del estudio de los relatos de vida se pudo comprender, por un lado, cómo implementaron acciones que modificaron los estilos de vida; por otro, los aprendizajes sobre cómo llevar, cuidarse y protegerse de la mejor manera antes, durante y después de la enfermedad.

Esta investigación se realizó en tres instituciones de salud de Medellín con NNA en condición de enfermedad: Hospital Infantil Concejo de Medellín, Clínica Las Américas y Clínica Universitaria Bolivariana. Estas instituciones han adecuado sus espacios, para que los NNA que asisten allí, por un diagnóstico o condición de enfermedad, se les ofrezca un acompañamiento educativo-pedagógico.

Como parte del desarrollo del proyecto, se determinaron conceptos tales como: infancia, familias, prácticas de cuidado y ECNT. La infancia, según Jaramillo (2007) y Casas (2006), tiene un carácter histórico, cultural y social que ha tenido diferentes transformaciones a lo largo del tiempo y, por lo tanto, se plantea la necesidad de reconocer la pluralidad de infancias. Por su parte, la familia, de acuerdo con Oliva y Villa (2014), es la unidad principal de las sociedades; su estructura ha variado en cuanto a sus funciones y composiciones: la nuclear está compuesta por dos adultos, hombre y mujer con sus hijos; la extendida está integrada por padres, hijos, abuelos y otros familiares; la monoparental es aquella en la que los hijos viven solamente con uno de sus padres. 
A su vez, las prácticas de cuidado, tal como lo plantea Amar (2015) y la Organización de Estados Iberoamericanos (OEI) (2018), son acciones que se basan en las creencias, costumbres y hábitos que los miembros de cada sociedad llevan a cabo con sus hijos, con el fin de promover aprendizajes; la antropología permite comprender cómo desde cada cultura, se distingue lo que las personas sienten, piensan y hacen en relación con la propia atención en salud (Muñoz y Vásquez, 2007). Por último, las ECNT, desde los planteamientos de Moiso (2007) y la Organización Mundial de la Salud (OMS) (2018), son padecimientos de larga duración, entre los que se encuentran las enfermedades cardiovasculares y respiratorias crónicas, el cáncer y la diabetes. Según Moiso (2007),

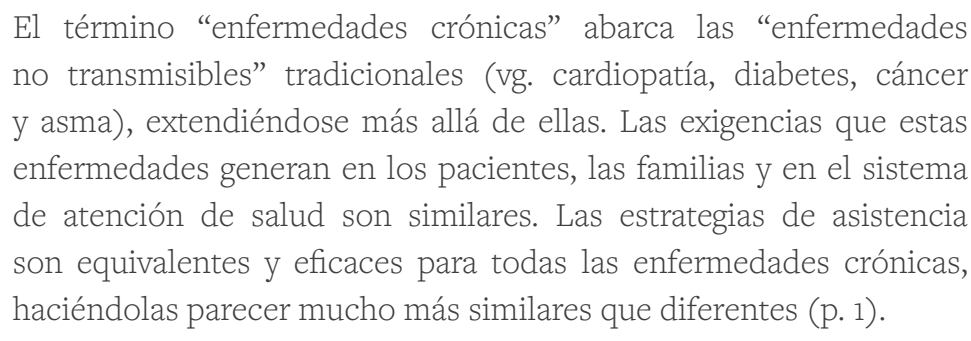

La ruta metodológica se estructuró desde dos componentes: por un lado, la investigación cualitativa, con enfoque narrativo; en este caso, la técnica utilizada fue la de los relatos de vida de NNA y cuidadores. Por otro, la propuesta pedagógica se sustentó desde el taller, cuyas actividades rectoras: juego y arte, se registraron en diarios pedagógicos. Los datos se sistematizaron en una matriz de análisis a partir de unidades de sentido, pre-categorías y categorías emergentes: emociones, juego y experiencias de aprendizaje; proceso de cuidado durante la enfermedad; hábitos de cuidado y autocuidado durante la enfermedad; y aprendizajes respecto al cuidado ante la enfermedad.

Esta investigación permitió llegar a la conclusión de que las prácticas de cuidado implementadas por las familias de NNA fueron pertinentes, pues se evidenciaron cambios en las rutinas cotidianas; estas prácticas se adaptaron a la experiencia de la enfermedad, con el fin de garantizar el bienestar de NNA, compartiendo las experiencias con otros desde el desarrollo de características resilientes. 


\title{
Metodología
}

La investigación cualitativa, desde una perspectiva interpretativa, permitió comprender la realidad de los NNA con ECNT, ya que produjo datos descriptivos tales como: "las propias palabras de las personas, habladas o escritas, y la conducta observable" (Quecedo y Castaño, 2002). Desde el enfoque narrativo, fue posible comprender: ¿quiénes son?, ¿cuál es el entorno? y ¿̇cuáles son sus realidades? Además de las relaciones interpersonales y comunicativas, al respecto Ricoeur afirma que:

\begin{abstract}
Las acciones adquieren un nuevo sentido narrativamente, uno mucho más coherente que si las tomamos por sí solas. Lo que se recompone es justamente su relación con el agente, con el "quién" del acto; la acción se convierte de este modo en "interacción”: Narrar es decir quién ha hecho qué, por qué y cómo, desplegando en el tiempo la conexión entre estos puntos de vista (Como se citó en Kosinski, 2015, p. 7).
\end{abstract}

La investigación permitió recoger los discursos de los sujetos a investigar, las percepciones y conductas observables que ofrecen descripciones de acontecimientos, interacciones, comportamientos e incluso pensamientos mediante la técnica de relatos de vida que hacen referencia a una situación vivida; la guía utilizada se dividió en tres aspectos: antes, durante y después de la enfermedad, cada uno con varios tópicos y preguntas orientadoras. Mediante la implementación de los diarios pedagógicos, se sistematizaron las actividades observadas y realizadas. Mediante la triangulación de datos de estas dos técnicas, se determinaron categorías emergentes sobre las cuales se realizó el proceso de discusión de los resultados.

El proyecto contó también con la implementación de una propuesta pedagógica y su enfoque fue el taller. Desde los planteamientos de Perdomo (s.f.), el taller "es un ambiente educativo en el cual la interacción con el conocimiento es también interactiva e intersubjetiva entre los participantes" (p. 1). Desde el campo de conocimiento de la pedagogía hospitalaria se implementaron estrategias didácticas como el juego y el arte. El juego es la primera fuente de contacto del ser humano con su entorno social o familiar, el niño empieza a jugar desde el vientre con la madre como por instinto o necesidad biológica; es una actividad innata y placentera; además, se considera una estrategia importante para conducir al niño en el mundo del conocimiento y a establecer relaciones sociales con otras personas. El arte permite representar experiencias o deseos de diferentes maneras, a partir de los lenguajes expresivos. Según el Ministerio de Educación Nacional (MEN) (2014): 
El arte se hace presente en la vida de cada persona y se comparte de maneras diversas. Propicia la representación de la experiencia a través de símbolos que pueden ser verbales, corporales, sonoros, plásticos o visuales, entre otros. De esta manera, impulsar la exploración y expresión por medio de diversos lenguajes artísticos para encontrar aquello que no solo hace únicos a los individuos, sino que los conecta con una colectividad, resulta fundamental en la primera infancia, puesto que lleva a establecer numerosas conexiones: con uno mismo, con los demás, con el contexto y con la cultura (p. 13).

La población participante de la investigación fueron NNA, entre el primer mes de vida hasta los 15 años, en condición de enfermedad, concretamente con ECNT; los hospitales y clínicas en los que se llevaron a cabo los estudios fueron: Hospital Infantil Concejo de Medellín, Clínica Las Américas y Clínica Universitaria Bolivariana, instituciones ubicadas en la ciudad de Medellín.

También se utilizaron consentimientos informados para el trabajo con los NNA, padres o cuidadores y registros fotográficos y de voz. Para la sistematización se diseñó una matriz de Excel, en la cual se organizaron los datos que posteriormente permitieron extraer las unidades de sentido, pre-categorías y categorías, que dieron como resultado las categorías emergentes.

\section{Resultados y discusión}

Para la discusión de resultados se definieron cuatro categorías; dos de ellas surgieron de las mediaciones pedagógicas realizadas con base en la propuesta pedagógica: emociones, juego y experiencias de aprendizaje y procesos de cuidado durante la enfermedad; las otras dos surgen de la sistematización de los datos de los relatos de vida de NNA y cuidadores: hábitos de cuidado y autocuidado durante la enfermedad y aprendizajes respecto a cuidados ante la enfermedad.

\section{Emociones, juego y experiencias de aprendizaje}

Estar en un contexto hospitalario no es una experiencia agradable; es una situación compleja que tiene un impacto social, que afecta tanto a quien la padece como a su familia. Por esta razón, en esta investigación se buscó implementar estrategias didácticas basadas en el juego y el arte, que permitieran evidenciar cambios en los sujetos al momento de comunicarse, expresar sus emociones y relacionarse 
con los demás en el contexto hospitalario. Además, estas estrategias permitieron potenciar y fortalecer los procesos de enseñanza y aprendizaje en cuanto a la enfermedad, sus cuidados y síntomas, en pro de mediar en estos, para favorecer la construcción e identificación de autoesquemas, el fortalecimiento de relaciones intra e interpersonales y las relaciones familiares.

Así, el juego se determina como estrategia de mediación pedagógica; de acuerdo con Damián (2014), “[...] el juego supone un medio esencial de interacción con los iguales y, sobre todo, induce al descubrimiento de nuevos sentimientos, sensaciones, emociones y deseos que van a estar presentes en muchos momentos del ciclo vital” (p. 121). Como lo menciona este autor, es importante generar estos espacios, que mediados por el juego y el arte permiten la participación de NNA y los cambios en el contexto hospitalario, para que este se convierta en un espacio de alegría y aprendizaje.

Algunas de las actividades que se llevaron a cabo fueron: "protejo mi cuerpo", “monstruo de las emociones", “medalla para valientes”, “escudo protector”, entre otras. Mediante estas experiencias se establecieron distintas relaciones con las demás personas, lo que ayudó a que desarrollaran su imaginación, a relacionarse con otros niños, a que tuvieran más confianza al comunicarse, jugar, distraerse y aprender. Con la implementación de esta propuesta, se evidenció que el juego y las relaciones sociales son parte fundamental del desarrollo y crecimiento de los NNA. Algunos de los testimonios recolectados en el desarrollo de estas estrategias fueron:

- Cuidador de niña de cinco años: "las actividades permiten que la imaginación, sueños y pensamientos de nuestros hijos sean coadyuvantes en la recuperación. Nos permiten a nosotros como padres ver sus caritas de nuevos con una sonrisa. Gracias” (Comunicación personal, 2019).

- Cuidador de niño de ocho años: "las actividades me parecen muy apropiadas para los niños, los sacan de la rutina del hospital y les ayudan a aprender el cuidado que deben tener con su cuerpo y la importancia de reconocer sus emociones. Gracias” (Comunicación personal, 2019).

- Niña de 12 años con cáncer: "las actividades me han parecido muy relajantes, entretenidas, ayudan a los niños a olvidarse del hospital y su enfermedad por un rato y nos mantiene alegres" (Comunicación personal, 2019).

Las acciones realizadas estaban pensadas para los NNA de diferentes edades, con distintas enfermedades tales como: asma, diabetes, cáncer, entre otras, lo que permitió que el contexto hospitalario se convirtiera en un espacio inclusivo y agradable, propicio para fortalecer las habilidades sociales. Blanco (2009) afirma que: “[...] las habilidades sociales son el arte de relacionarse con las demás personas y el mundo que nos rodea. Son conductas destinadas a conseguir un objetivo, defender nuestros derechos, ser asertivos en la expresión de nuestras emociones y deseos” (s.p.). 
En la implementación de la propuesta, se logró fortalecer las relaciones interpersonales entre los NNA y sus cuidadores, con el fin de promover experiencias positivas para el aprendizaje y la participación en un contexto diferente, donde se crean y fortalecen las relaciones sociales, pues como lo menciona Blanco (2009), son fundamentales en el bienestar de los NNA en condición de enfermedad y es más favorable para su recuperación. Un cuidador de una niña de diez años expresa:

En lo personal me agradan mucho las actividades que hacen con los niños, se distraen un poco y comparten con los demás niños, es una labor muy hermosa al hacer sonreír y alegrarles un poco el paso por el hospital a los niños (Comunicación personal, 2019).

Los espacios generados en cada actividad permitieron transformar el contexto hospitalario, generando un clima más favorable para el aprendizaje de los niños y los adolescentes, integrando a la familia al proceso de adaptación. Permitieron que cada persona que se encontraba en las habitaciones cambiara su estado de ánimo, compartiera con otros que estaban a su alrededor, se distrajeran un poco y aprendieran a diferenciar y expresar sus emociones. Al respecto, Filella menciona que: “[...] la educación emocional debe ser considerada como un proceso educativo continuo y permanente, que pretende potenciar el desarrollo emocional como complemento indispensable del cognitivo. Ambos elementos son esenciales para el desarrollo de la personalidad integral" (Como se citó en Oros et al., 2011, p. 496).

Mediante la actividad "el monstruo de las emociones" se pudo evidenciar que los NNA al estar en el contexto hospitalario, sintieron todas las emociones; algunas de las expresiones manifestadas fueron: "todas las emociones, porque no me gusta el hospital” (Comunicación personal, niña de cinco años, 2019); “a mí me gusta el hospital porque parece un hotel de los de la playa, pero no me gusta cuando me chuzan o me lastiman, por eso pongo todas las emociones" (Comunicación personal, niño de ocho años, 2019).

Además, se observó que, al momento de realizar las actividades propuestas, los niños se veían felices, pero algunos cuidadores manifestaban que en la habitación se aburrían, lloraban, se ponían muy tristes y solo se tranquilizaban cuando hacían alguna actividad que les llamara la atención; sentían alegría al compartir con los demás niños, y miedo cuando las enfermeras entraban a la habitación.

Finalmente, cabe resaltar que para transformar los contextos hospitalarios es necesario determinar cuáles son los efectos negativos de la hospitalización en cuanto a las relaciones sociales, lo que ayudaría a diseñar estrategias que motiven a la participación de los NNA en cada una de las actividades, en las que logran experimentar sensaciones diferentes y descubren capacidades o habilidades que no sabían que tenían; de esta manera, las experiencias de aprendizaje serán significativas. 


\section{Procesos de cuidado durante la enfermedad}

Los cuidados que brindan los padres, cuidadores y personal médico a los NNA durante la enfermedad son pertinentes para su diagnóstico, tratamiento y estancia en las instituciones de salud. Los padres y cuidadores desempeñan un rol importante cuando incentivan a los NNA con prácticas de cuidado que deben tener, a partir de su enfermedad e ir implementado con ellos los cambios que se presenten en sus rutinas diarias, como por ejemplo: modificación de la hora de levantarse, ya que algunos deben tomar medicamentos; o cuando están en el hospital deben despertarse temprano para que el médico efectué la revisión; en algunas ocasiones, se cambia el momento de tomar el baño, si antes lo hacían solos, ahora lo deben hacer acompañados, o por el contrario, si antes se bañaban con agua fría, ahora lo deben hacer con agua caliente; asimismo se presentan modificaciones en la dieta alimentaria, dependiendo del tipo de enfermedad que presenten.

En los hábitos cotidianos, los NNA que realizaban algún deporte, si su enfermedad se lo impide, no pueden volver a practicarlo; otros deben quedarse en sus casas, ya que no pueden salir si está haciendo mucho frío o mucho calor; algunos deben permanecer en sus hogares si tienen las defensas muy bajas, para evitar posibles complicaciones en su tratamiento; unos cuantos deben dejar de asistir a la escuela, porque su estadía permanente en el hospital no les permite seguir con el proceso. También se presentan cambios en su aspecto físico, por ejemplo: bajar de peso, pérdida de cabello e inflamaciones en alguna parte del cuerpo.

La actividad "protejo mi cuerpo" fue muy enriquecedora, porque se notó el compromiso y dedicación tanto de los niños como de los padres por el cuidado del cuerpo, por mantenerlo sano, limpio y en las mejores condiciones; durante el desarrollo, los participantes decidieron contar sus experiencias tanto positivas como negativas, pues no todas las familias contaron al resto de grupo que habían escrito en el escudo protector, pero aun así se llegó a una conclusión y aprendizaje de lo que se debe o no hacer con NNA y enseñar siempre que se debe trabajar por su bienestar físico y psicológico. Con base en estos planteamientos, Falleiros et al. (2014) establecen que

La necesidad de relaciones sustentadoras continua se refiere a la presencia del cuidador del niño y al modo de interacción constante con él, por medio de cuidados físicos e interacciones afectivas. La necesidad de protección física y seguridad objetiva garantizar condiciones favorables para la manutención de la integridad física y fisiológica del niño, en la que participan: alimentación, higiene, sueño, protección, movimientos, acompañamiento del crecimiento y desarrollo, apoyo a los hábitos saludables, y protección contra 
infecciones y accidentes, así como la reglamentación con base en legislación y otras medidas que protejan al niño de daños físicos, sociales y ambientales (p. 605).

Se evidenció también que los padres brindan apoyo y cuidado en todo el proceso de la enfermedad; comienzan a informarse, a investigar y a preguntar al personal de salud sobre los padecimientos de sus hijos o hijas y cómo deben llevarlos; se muestran interesados en saber cómo sobrellevarlos de la mejor manera y con los cuidados pertinentes.

Al inicio de la enfermedad los padres se muestran con preocupación y tristeza, pero cuando empiezan a convivir con el padecimiento de sus hijos, intentan manejarlo de la mejor forma posible, cambiando las rutinas y los hábitos alimenticios; inician un proceso en en el que se preocupan por el bienestar de sus descendientes y, en todo momento, tratan de evitar sucesos que afecten su salud.

En este proceso de cuidado durante la enfermedad, algunas familias se vuelven más fuertes; mientras que, por el contrario, otras más frágiles, pues lo que esperan durante esta experiencia de enfermedad y tratamiento es una pronta recuperación y regresar a la vida como acostumbraban a llevarla, generando un sentimiento de ansiedad. En este proceso de cuidado, durante el padecimiento, es fundamental hablar con los padres y explicarles que va a ser un tiempo de ajustes continuos.

Con la realización de la actividad “el monstruo de las emociones”, se evidenció que los niños, niñas, cuidadores y padres aprendieron a diferenciar y expresar sus emociones a partir del monstruo de colores. Estaban felices con sus monstruos, algunos empezaron a poner el azul, otros el amarillo o el rojo; al final, al observar las creaciones de todos, el monstruo quedó de todos los colores. A los participantes se les preguntó que emoción le habían puesto a su monstruo y decían: "todos los colores".

$\mathrm{Al}$ respecto, Oros et al. (2011) mencionan que la educación emocional debe ser considerada como un proceso educativo continuo y permanente, que pretende potenciar el desarrollo emocional como complemento indispensable del cognitivo. Ambos elementos son esenciales para el desarrollo de la personalidad integral. Las emociones influyen en las decisiones que tomamos a lo largo de la vida, se manifiestan en expresiones, ya sean faciales o corporales; por esto es importante saberlas maneja.

Los cuidados de los NNA varían dependiendo de su enfermedad; por ejemplo, los niños con algún tipo de cáncer y que están en el proceso de quimioterapia, deben cuidarse al tener contacto con personas con virus o enfermedades contagiosas, porque durante este proceso sus defensas son muy bajas; aquellos con enfermedades respiratorias crónicas deben cuidarse de los climas fríos, de los lugares en donde haya mucho polvo o donde trabajen con sustancias químicas que afecten su respiración, tampoco pueden tener mascotas; los que padecen enfermedades cardiovasculares deben abstenerse de realizar actividades que impliquen agotamiento físico; y los que tienen diabetes, deben cambiar sus rutinas alimenticias omitiendo los azúcares. 
En la experiencia se evidenció que los cuidados que se dan a los NNA por parte de sus padres son oportunos y pertinentes, pues los que presentan enfermedades agudas tienen una pronta recuperación y aquellos que presentan enfermedades crónicas, aprenden y comienzan a adaptarse a los cambios que deben hacer para mantenerse en un buen estado de salud. Por tal motivo, se realizaron diferentes tipos de actividades, enfocados en la identificación de los aspectos propios de la enfermedad, con los cuales se buscaba que las personas mejoraran prácticas de cuidado para que fuera más fácil establecer signos o síntomas de alarma, cambios en rutinas, entre otros aspectos directamente relacionados con la enfermedad. Durante las entrevistas a NNA, padres y cuidadores surgieron algunos cuidados que ellos practican durante la enfermedad, tales como:

- "Los niños con las defensas bajas no pueden jugar con otros niños" (Comunicación personal, 2019).

- "Siempre hay que estar pendientes dándole vitaminas, puesto que ellos dejan de comer mucho” (Comunicación personal, 2019).

- “Cancelación del estudio, les toca salirse de estudiar y también, en algunas ocasiones, cancelar las salidas” (Comunicación personal, 2019).

- "Evitar que el niño o niña se serene, evitar que tome cosas frías y cambiar constantemente las cortinas y las sábanas” (Comunicación personal, 2019).

Las prácticas de cuidado que se les brinda a los NNA durante la enfermedad son pertinentes, pues los padres aprenden cómo deben llevarla y asimilan muy bien los cambios; se puede decir que adoptan una nueva vida, donde todo cambia: los hábitos de higiene y alimentación, así como las rutinas diarias. Los padres además de adaptarse a la enfermedad ayudan y enseñan a sus NNA cuáles deben ser las atenciones mientras están en un tratamiento o, por el contrario, los cambios que se deben implementar de por vida. En conclusión, los cuidados durante la enfermedad son muy importantes, pues facilitan el proceso de una pronta recuperación y una manera de llevar la enfermedad de buena manera, de tal forma que no empeoren.

\section{Hábitos de cuidado y autocuidado respecto a la enfermedad}

Durante la implementación del proyecto se realizó una recolección de datos mediante narrativas, teniendo en cuenta la experiencia vivida por los NNA; acción que permitió evidenciar los hábitos de cuidado y autocuidado durante la enfermedad. Según la OEI (2018), este tipo de hábitos 
hacen parte de la cotidianidad [...] son de vital importancia en la promoción del crecer bien, desarrollo y aprendizaje de las niñas, los niños al generar bienestar y enriquecer las experiencias pedagógicas [...]. Cuidar implica reconocer, valorar, respetar, acoger y atender de manera sensible y oportuna los llamados, requerimientos y necesidades de niñas, niños (p. 8).

Mientras que el autocuidado, de acuerdo con Prado et al. (2014), se entiende como:

[...] el conjunto de acciones intencionadas que realiza la persona para controlar los factores internos o externos, que pueden comprometer su vida y desarrollo posterior. El autocuidado, por tanto, es una conducta que realiza o debería realizar la persona para sí misma [...] con el interés de mantener un funcionamiento vivo y sano, continuar, además, con el desarrollo personal y el bienestar (p. 838).

Teniendo en cuenta lo planteado anteriormente por los autores y los datos obtenidos en las narrativas, los hábitos de cuidado y autocuidado se deben inculcar desde temprana edad en los NNA, porque a medida que van creciendo, se vuelven parte de su rutina diaria.

En los relatos de vida, las familias resaltan su papel fundamental en la adquisición de este tipo de hábitos durante la crianza de los NNA; las conductas aprendidas tienen que ver con las orientaciones brindadas por ellos, en las que implementaron prácticas de higiene, alimentación y hábitos, tales como tomar los medicamentos diariamente, con la idea de mejorar el cuidado del cuerpo y la salud. De acuerdo con el Ministerio de Salud (2018): “[...] el padecer ECNT afecta de forma considerable la calidad de vida de la persona debido a las adaptaciones que deberá hacer según el tipo de enfermedad" (s.p.).

Los hábitos de cuidado y autocuidado involucran prácticas saludables que ayudan a los sujetos a sentirse bien; las familias y los niños se adaptan a las características y fases de cada enfermedad e implementan las recomendaciones de los médicos. Aunque el cuidador de un niño de ocho años reconoce que: "al principio de la enfermedad lo querían tener en una urna de cristal” (Comunicación personal, 2019); de igual manera, menciona realizar o implementar cuidados especiales, teniendo en cuenta la enfermedad.

Para los NNA con asma se debe prestar más atención al aseo de la casa, no pueden estar donde alguien está fumando, deben evitar el sereno y tomar cosas frías, tratar de cambiar constantemente las cortinas, las sábanas para evitar que les dé alergia y estar pendiente siempre por si les da gripe, ya que es una alarma instantánea. $\mathrm{Al}$ respecto, una niña de siete años expresa que: 


\begin{abstract}
mi rutina es un puf cada vez que me siento mal y siempre que me va a bañar un baño caliente, pues yo como de todo. Haciéndole caso a lo que me dice mi papá y los medicamentos que necesito para cuidarme (Comunicación personal, 2019).
\end{abstract}

En cuanto a la diabetes se recomienda cambiar la alimentación: la comida debe ser saludable, no consumir gaseosas, dulces, entre otros. Además, los padres y cuidadores deben estar pendientes de las aplicaciones de la insulina, caminar mucho. Al respecto un niño de 10 años manifiesta que: "me toca consumir alimentos sin azúcar, también sin grasa y estarme aplicando la insulina" (Comunicación personal, 2019).

En relación con los cuidados de los niños con cáncer, como "mantienen las defensas bajitas" es importante que no salgan, que no jueguen con otros niños en ese tiempo. Un cuidador de una niña de dos años refiere que: "ellos dejan de comer mucho, entonces hay que estar pendientes dándole vitaminas", “evitar que tomen cosas del suelo porque les puede dar alguna bacteria” (Comunicación personal, 2019).

Y finalmente en las enfermedades cardiovasculares es importante adaptarse al tipo de alimentación que le recete el nutricionista, estar pendiente de los medicamentos, tomarlos todos los días, caminar, subir escaleras y evitar el sedentarismo.

La atención que se debe brindar a los NNA con ECNT requiere dedicación, cuidados individuales y permanentes para evitar recaídas u hospitalizaciones. Aunque los hábitos de cuidado y autocuidado parezcan ser algo simple, en realidad involucra reconocer la identidad de cómo cuidar su propio cuerpo y el del otro, adquiriendo hábitos nuevos en sus rutinas.

Durante la implementación del proyecto se logró evidenciar que las ECNT en los NNA es algo que involucra a toda la familia, puesto que la hospitalización y los tratamientos son un gran reto para la misma, pues hacen todo lo posible para brindar los cuidados que necesitan sus hijos. Las familias aprenden a adaptarse a las características de cada enfermedad y sus diferentes fases; pero todo esto causa alteraciones en las rutinas de todos sus integrantes, ya sea en el trabajo, escuela, universidad, tiempo libre, entre otros. Algunos de los cambios que surgieron en las rutinas de los cuidadores por la enfermedad de sus hijos fueron:

- Cuidadora de niña de dos años con diagnóstico de cáncer: “yo la tenía en ese programa de crecimiento y desarrollo, la tenía en estimulación y en piscina, ya tuve que retirarla" (Comunicación personal, 2019).

- Cuidadora de niña de tres años con diagnóstico de cáncer: "me retiré del estudio, me tocó salirme de estudiar, ya se empezaron a cancelar las salidas con la niña porque salía mucho con ella” (Comunicación personal, 2019). 
Sufrir una ECNT conlleva a un cambio o alteración en la vida cotidiana de todas las personas alrededor de quien la padece, además de esfuerzos familiares con el fin de garantizar la calidad de los cuidados antes, durante y después de la enfermedad.

\section{Aprendizajes respecto a cuidados ante la enfermedad}

La sistematización de la información obtenida de los relatos de vida de NNA y cuidadores permitió evidenciar diferentes aprendizajes en relación con las prácticas de cuidado, rutinas, entre otros aspectos. Al respecto, Gagné establece que

El aprendizaje es un proceso de cambios en la capacidad o conducta de los organismos vivos, más o menos estables y que persiste pese al tiempo transcurrido, y que no puede ser explicado sencillamente por procesos de crecimiento o maduración (procesos internos), ni es producto de estados patológicos, debe ser resultado de la interacción de su organismo con su medio externo. Es decir el aprendizaje es un proceso y un producto (resultados), que pone énfasis en la naturaleza de los procesos internos, en las situaciones ambientales o eventos externos, en el tipo de conductas que pueden ser modificadas mediante el aprendizaje y las características que resultan del mismo (Gonzáles et al., 2006, p. 5).

Tomando como base lo anterior, y a partir de los relatos de vida tanto de NNA como de cuidadores en los diferentes contextos hospitalarios, se pudo evidenciar que ellos construyeron diversos aprendizajes a partir de las experiencias que viven cotidianamente en relación con la condición de enfermedad. Cabe destacar que estos aprendizajes no se evidencian de un día para otro, puesto que involucran diferentes procesos individuales e incluso familiares. Por ejemplo, algunas personas manifestaron que el hecho de ir al hospital ya es parte de su cotidianidad; en cambio, otros dicen que es complejo el tener que estar movilizándose a la institución de salud, porque no es habitual que un familiar se enferme y que tengan que adaptar su rutina para poder acompañarlo y prestarle la atención necesaria.

Los NNA y cuidadores construyen diferentes aprendizajes a partir de las experiencias vividas, en este caso a partir de la enfermedad, los cuales van más enfocados a los cuidados que se deben tener para sobrellevar la situación, ya sea por parte de los mismos NNA como por la familia, puesto que se presentan casos en los que se evidencian unas prácticas de cuidado que han dado buenos resultados y otras no tanto; a partir de esto, las personas optan por buscar más información para mejorar esas prácticas, aprendiendo de lo realizado; tanto así que luego lo vuelven a poner en práctica con otras 
personas, contándoles lo que hacían, lo que les funcionó y lo que no les dio buen resultado para que otros decidan si también ponen en práctica estas recomendaciones o toman las que consideran útiles y las adaptan a sus contextos.

A partir de los aprendizajes obtenidos, las personas cambian algunos aspectos de su vida y su cotidianidad para que el proceso de la enfermedad sea más llevadero y comprensible, puesto que los cambios que surgen a nivel físico, emocional y psicológico son en la mayoría evidentes y otros no tanto, por lo que se realizan diferentes tipos de actividades enfocadas en la identificación de los aspectos propios de la enfermedad, con los cuales se busca que las personas mejoren prácticas de cuidado que implementen para que sea más fácil identificar signos o síntomas de alarma, cambios en rutinas, entre otros aspectos directamente relacionados con la enfermedad. Algunos de los aprendizajes recolectados a partir de los relatos de vida fueron:

- Cuidador de niño con diabetes: “[...] he aprendido que hay que estar pendiente de los de uno y que no se le puede pasar a uno por lo mínimo que sea, no se le puede pasar a uno de sus hijos enfermedades [...] he aprendido a conocer nuevas cosas como lo de la diabetes que no sabía nada nada y he aprendido muchas cositas como la aplicación de la insulina" (Comunicación personal, 2019).

- Cuidadora de niña de dos años: “[...] lo que pasa es que uno ya ve las cosas de diferente manera, la vida ya es otro cueno [...] se vuelve uno más humano, ya lo material y lo que es en sí lo físico pasa a un segundo plano, lo más importante definitivamente es la salud" (Comunicación personal, 2019).

- Niño de 12 años: "yo debo de llevar mi enfermedad con mucha paciencia, no debo desesperarme ni darme rabias ni preocuparme" (Comunicación personal, 2019).

- Cuidadora niña de tres años: “que cuando uno está bien se aleja mucho de Dios, solo lo busca en las cosas malas y es donde más se une la familia” (Comunicación personal, 2019).

Este tipo de aprendizajes se manifesta en las personas de diferentes maneras. En ocasiones se evidencian casi inmediatamente luego que ocurre una situación que altera la cotidianidad; otras veces se tarda un poco más debido a que viven un proceso de duelo y de aceptación de la situación, ya que el estilo de vida se ve afectado. Los aprendizajes se evidencian con mayor facilidad cuando las personas son colocadas a prueba, ya sea siguiendo recomendaciones brindadas por el personal de salud que lleva el caso del niño, ampliando la información que se le brinda y haciendo preguntas al respecto, implementando nuevas prácticas de cuidado o simplemente acompañando a los pacientes durante la enfermedad. 
En este punto influye mucho la motivación de las personas para superar procesos que pueden ser adversos y el interés que tengan en continuar adelante, según la realidad y el contexto que afronten; puesto que hay factores que inciden directa o indirectamente en los diferentes aspectos de su vida: personales, familiares y comunitarios; factores que pueden llegar a ser de riesgo o de protección, los cuales dejan diferentes tipos de aprendizaje en la persona que los experimente.

\section{Conclusiones}

La investigación permitió adquirir nuevas experiencias mediante la implementación de mediaciones pedagógicas en los diferentes contextos hospitalarios; favoreció los procesos de enseñanza y aprendizaje tanto de los NNA y cuidadores como de las docentes. Se propició un ambiente diferente que posibilitó que el proceso de hospitalización fuera más ameno y, al mismo tiempo, que tuviera experiencias significativas que los ayudara a mejorar sus prácticas de cuidado en cuanto a la alimentación, higiene, hábitos, ejercicio, entre otros; acciones que no solo estaban diseñados para la estancia hospitalaria, sino para que se continuaran implementando en los hogares.

También se logró llevar a cabo estrategias didácticas que motivaran a los NNA a mejorar su salud; se lograron fortalecer los lazos familiares, además de promover el bienestar en los niños mediante experiencias que les posibilitaron relacionarse con los demás, experimentar emociones y explorar su creatividad, lo que permitió que los NNA vieran el hospital como un lugar mucho más agradable, que ofrece oportunidades para divertirse, jugar y aprender.

Finalmente, es pertinente resaltar que los diferentes elementos utilizados durante las mediaciones pedagógicas en los contextos hospitalarios, como las actividades de la propuesta pedagógica y la guía para los relatos de vida para la identificación y descripción de las prácticas de cuidado que implementan los NNA con ECNT y sus familias, son adecuadas porque permiten que este proceso se realice de una forma más amigable, que no interfiere de forma abrupta en la vida de los NNA hospitalizados, sino que por el contrario les facilitara un poco sobrellevar la situación por la que atraviesan, aprendiendo más de sí mismos, de sus familias y de quienes les rodean. 


\section{Referencias}

Amar, J. (2015). Desarrollo infantil y prácticas de cuidado. Editorial Universidad del Norte.

Blanco, E. (2018). ¿Qué son las habilidades sociales? Tipos y para qué sirven. https:// psicologosoviedo.com/especialidades/ansiedad/habilidades-sociales/

Casas, F. (2006). Infancia y representaciones sociales. Política y Sociedad, 43(1), 27-42. https://revistas.ucm.es/index.php/POSO/article/view/23779

Damián, M. (2014). Contextos, juegos y expresión de las emociones de los escolares: su importancia en el desarrollo psicológico y en la educación. http://revistarayuela.ednica. org.mx/sites/default/files/121-130.pdf

Falleiros, D., Pereira, N., Pancien, L., Ramallo, M., Pamplona, V., y Malone, M. (2014). La seguridad del niño en la perspectiva de las necesidades esenciales. Revista LatinoAm. Enfermagem, 22(4), 604-610. https://www.scielo.br/pdf/rlae/v22n4/es_01041169-rlae-22-04-00604.pdf

Gonzáles, E., Campos, J., y Palomino, J. (2006). Escuela Académica Profesional de Psicología. Introducción a la psicología del aprendizaje. Doi: 978-9972-34-540-1

Jaramillo, L. (2007). Concepciones de infancia. Zona próxima, 8, 108-123. http://www. redalyc.org/articulo.oa?id $=85300809$

Kosinski, A. (2015). Una manera de responder ¿quién soy?: la identidad narrativa de Paul Ricoeur. Revista del Departamento de Filosofía, 213-221. http://revistas.filo.uba.ar/ index.php/avatares/article/view/322/325

Ministerio de Educación Nacional. (2014). Documento 21: El arte en la educación inicial. Serie de orientaciones pedagógicas para la educación inicial en el marco de la atención integral. https://www.mineducacion.gov.co/1759/articles-341880_archivo_pdf_doc_21.pdf

Ministerio de Salud. (2018). Enfermedades crónicas no transmisibles - Comunidad. http:// www.rionegro.gov.ar/index.php?contID $=17803$

Moiso, A. (2007). Enfermedades Crónicas No Transmisibles: el desafío del siglo XXI. En H. Barragán (Dir.), Fundamentos de Salud Pública (pp. 265-290). Universidad Nacional de La Plata.

Muñoz, L., y Vásquez, M. (2007). Mirando el cuidado cultural desde la óptica de Leininger. Colombia Médica, 38(4), 98-104. http://www.scielo.org.co/pdf/cm/v38n4s2/ v38n4s2a11.pdf

Oliva, E., y Villa, V. (2014). Hacia un concepto interdisciplinario de la familia en la globalización. Justicia Juris, 10(1), 11-20. http://www.scielo.org.co/pdf/jusju/vion1/ v1on1ao2.pdf 
Organización de Estados Iberoamericanos. (OEI). (2018). Prácticas de cuidado y crianza. https://redes.colombiaaprende.edu.co/ntg/men/pdf/Practicas_de_Cuidado.pdf

Organización Mundial de la Salud. (OMS). (2018). Organización Mundial de la Salud.

Oros, L., Manucci, V., y Richaud, M. (2011). Desarrollo de emociones positivas en la niñez. Lineamientos para la intervención escolar. Educación y Educadores, 14(3), 493-509. https://www.redalyc.org/pdf/834/83422605004.pdf

Perdomo, N. (s.f.). Los retos del aprendizaje: El taller como estrategia pedagógica. https:// www.emagister.com/blog/los-retos-del-aprendizaje-el-taller-como-estrategiapedagogica/\#: :text=Seg\%C3\%BAn\%2oVasco\%2C\%2oun\%2otaller\%20 \%E2\%80\%9C\%E2\%80\%A6,un\%20ambiente\%2orico\%2oen\%2orecursos

Prado, L., González, M., Paz, N., y Romero, K. (2014). La teoría Déficit de autocuidado: Dorothea Orem punto de partida para calidad en la atención. Revista Médica Electrónica, 36(6). http://scielo.sld.cu/scielo.php?script=sci_ arttext\&pid=S1684-18242014000600004\#: :text=E1\%20concepto\%20 b\%C3\%A1sico\%2odesarrollado\%2opor,su\%2ovida\%2oy\%2odesarrollo\%20 posterior

Quecedo, R., y Castaño, C. (2002). Introducción a la metodología de investigación cualitativa. Revista de Psicodidáctica, 14, 5-39. 


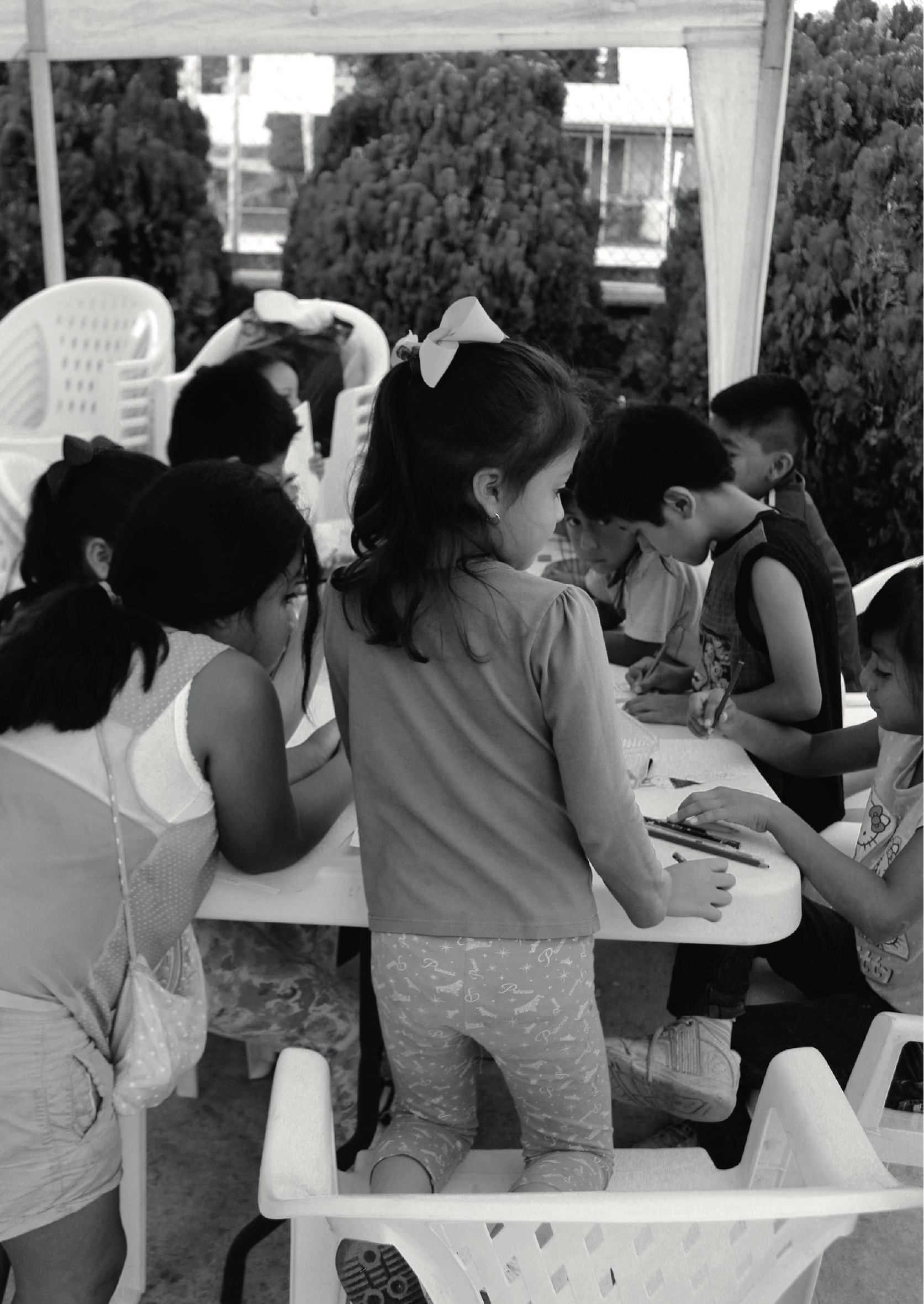

